\title{
Nécessité et refus de l'image dans la poésie de Philippe Jaccottet
} Evelio Miñano

\section{Citer ce document / Cite this document :}

Miñano Evelio. Nécessité et refus de l'image dans la poésie de Philippe Jaccottet. In: Littératures 17, automne 1987. pp. 161171 ;

https://www.persee.fr/doc/litts_0563-9751_1987_num_17_1_1419

Fichier pdf généré le 29/03/2018 


\section{Nécessité et refus de l'image dans la poésie de Philippe Jaccottet}

Il est évident que l'image constitue un des centres d'inquiétude de l'œuvre de Jaccottet, ne serait-ce que par la fréquence avec laquelle il prend des positions déterminées ou émet des jugements à ce sujet dans ses ouvrages. Cette attitude critique correspond parfaitement à la mise en question sur laquelle est bâtie une grande partie de la poésie contemporaine. Effectivement, un double questionnement du langage et de l'entreprise poétique sous-tend cette œuvre, et à un point tel qu'il s'établit des rapports étroits entre les positions complexes que prend le poète vis-à-vis de l'image et la façon dont il conçoit, puis juge par moments, son univers particulier. Si l'image apparaît parfois ouvertement refusée ou, au contraire, requise, il ne s'agit pas seulement d'un fait de rhétorique mais d'un élément essentiel à une entreprise poétique particulière, car cette nécessité et ce refus sont enracinés dans un double désir de s'élever vers l'Absolu et de ne pas effacer l'ici-bas. La poésie, et surtout l'image, étant les instruments privilégiés dans cette quête de l'Illimité qui se veut conciliée à une acceptation de la réalité matérielle, elles sont profondément marquées par les échecs ou les réussites d'une telle tentative, ainsi que par sa nature bipôlarisée à l'extrême. Et cela, comme nous le verrons, non seulement à travers une prise de position déterminée vis-à-vis de l'image, mais aussi par les caractères particuliers que prendront ses réalisations dans l'écriture, le tout montrant les liens profonds qui relient une conception et une pratique de l'image à l'univers poétique où celle-ci s'inscrit.

Tout d'abord, l'image est objet de refus ; telle est la conséquence du désir qu'a le poète de se réconcilier avec le monde de l'ici-bas, si imparfait soit-il, et de ne pas sombrer dans un élancement qui finirait par l'effacer. Elle peut, en effet, constituer un sérieux obstacle à la tentative d'assumer 
pleinement la condition des choses et voiler leur authentique nature en nous offrant un substitut imaginaire qui les embellisse démesurément. Dès les derniers poèmes de l'Effraie, et surtout dans les deux ouvrages suivants, L'Ignorant et Airs, Jaccottet a décidé de se soumettre en quelque sorte aux choses et d'être accueillant à l'appel qui peut se révéler dans certaines nuances. Ainsi, s'exclame-t-il :

«L'effacement soit ma façon de resplendir, la pauvreté surcharge de fruits notre table, la mort, prochaine ou vague selon son désir, soit l'aliment de la lumière inépuisable » (1).

L'entreprise du poète se résume dans cette tentative de faire jaillir de la mort " une lumière inépuisable ", un aliment éthéré pour l'être qui le fera participer simultanément aux limites et à l'Illimité, de trouver dans les imperfections et les signes douloureux de la réalité matérielle le passage qui la relie à son opposé. Et afin de recueillir fidèlement ces signes, il doit s'effacer au maximum, faire table rase de tout ce qui pourrait s'interposer entre lui et la réalité. Si les sentiments et l'anecdote personnels apparaissent de moins en moins au fur et à mesure que son œuvre avance, c'est parce qu'il compte se rapprocher des choses et rendre son regard plus pénétrant en se dépouillant en tant qu'individu. De même, il refuse tout lyrisme ou toute émotion qui pourrait troubler son regard :

« Moins nos larmes apparaîtront brouillant nos yeux

et nos personnes par la crainte garrotées, plus les regards iront s'éclaircissant et mieux les égarés verront les portes enterrées " (2).

Ainsi, dans la sérénité qui naît de l'acceptation, trouvera-t-on « les portes enterrées ", les lieux de transition entre l'ici-bas et l'Illimité. Le poète doit être un " ignorant ", un " appauvri ", en ce sens qu'il doit se présenter dépourvu de toutes idées et clichés reçus devant les choses, afin de devenir plus perméable à celles-ci. Jaccottet résume cela magistralement en quelques mots : "A partir de rien telle est ma loi ".

Comme conséquence de tout cela, apparaît l'idée que la parole elle-même peut être un obstacle pour le rapprochement fidèle des choses. Il s'établit alors une opposition entre regard et parole (comme s'ils s'excluaient foncièrement), qui menace l'existence du poème et risque de le couper net :

«Tais-toi : ce que tu allais dire

en couvrirait le bruit.

Ecoute seulement : l'huis s'est ouvert »(3).

Si le poème s'achève là où précisément « l'huis s'est ouvert ", c'est parce que le parti pris du silence constitue, en réalité, une tentative de préserver ces moments privilégiés et rares, où l'essor se déploie :

" Cependant, même à qui chemine à mon côté même à ce chant, je ne dirai ce qu'on devine dans l'amoureuse nuit. Ne faut-il pas plutôt 
laisser monter au mur le silencieux lierre de peur qu'un mot de trop ne sépare nos bouches et le monde merveilleux ne tombe en ruines $\gg(4)$.

Mais malgré tout cela, le poème naît, l'impuissance de la parole vis-à-vis de l'Illimité s'exprime par la parole même.

La menace de silence pour le poème se transforme en menace de nomination pure et simple, pour l'image. Le poète tente maintenant d'approcher les choses en les présentant sans ornements, sans fausses parures, telles qu'elles sont, tout simplement nommées. Il arrive à cette conclusion après avoir constaté que l'image est impuissante à rendre fidèlement ce qui l'a frappé dans les choses, le mystère qui s'y cache et l'attire :

"Sur le moment je n'ai noté que cela. Bien conscient qu'une fois de plus je bâtissais une réalité à côté de l'autre ou autour d'elle, qui en avait gardé quelques traits mais en cachait ou déformait d'autres et, de ce fait, découragé d'avance. M'avouant par moments que le seul mot de "pré ", ou mieux de " prairie " en dirait plus que ces recherches toujours menacées de préciosité » (5).

La tentation de la nomination pure et simple est d'autant plus compréhensible que le poète prétend s'élever à l'Illimité dans une acceptation pleine du monde matériel. D'autre part, le pouvoir de séduction de l'image est si fort qu'elle peut non seulement déformer l'appréhension des choses mais nous faire croire qu'on a aussi percé la réalité extérieure et atteint l'ordre de la transcendance :

"Pour peu qu'on cède à cette pente, il se produit un foisonnement de relations plus ou moins baroques entre les choses qui peut, à bon marché, faire croire que l'on a découvert lcs sccrc̀tes structures du monde, alors qu'on a simplement tiré le maximum d'effets de l'imprécision d'une expression " (6).

Les images sont des solutions faciles pour le poète lors de sa confrontation à l'appel qu'il ressent ; elles ouvrent de fausses voies à notre interprétation du réel et, de plus, déforment celui-ci. Tout cela a mené Jaccottet à rêver d'une poésie sans images, suprême aboutissement, à ses yeux, de l'art du Hai-ku japonais, dont il montre l'influence surtout dans Airs et L'Ignorant.

Refus donc de la parole et de l'image au double profit d'une approche plus perçante de la réalité et de l'appréhension de l'Illimité, comme s'il y avait une exclusion foncière entre la parole poétique et celui-ci.

Un paradoxe saute aux yeux si l'on confronte la théorie et la pratique : Jaccottet décrie l'image mais, par ses écrits, il montre qu'il n'a pu s'en passer. Refusant explicitement ce procédé, il y recourt constamment, quitte à se sentir ensuite la proie de sa mauvaise conscience : 
«Et quand je pense à la rivière, à ce que j'ai fait de cette rivière, j'éprouve une espèce de honte à l'avoir pareillement déformée " (7).

La fréquence avec laquelle les poèmes renoncent explicitement à l'image puis sont contraints de les utiliser, nous fait penser qu'il y a une nécessité, parallèle au contrepoids qui s'oppose à la menace du silence, de ne pas sombrer dans la nomination directe. En effet, le poète reconsidère son premier refus unilatéral de la métaphore au profit du simple dénombrement car, dans les deux cas, il constate une même impuissance à percer ce qui l'entoure :

«Refus de la métaphore par souci de ne pas trahir une plus mystérieuse simplicité.

Peupliers éclairés. Peupliers plus clairs, ensoleillés.

L'herbe quí s'assombrit sous les peupliers ensoleillés, de loin en loin, dans la vallée. Et rien n'est dit $"(8)$.

Cette conscience de l'échec n'empêche cependant pas que te poète réponde à l'appel de l'Illimité par l'image, tentant ainsi de mettre à jour l'inconnu, l'« autre ", auquel celui-ci renvoie. C'est consciemment qu'il utilise les images " comme des portes " pour aller vers ce qui le fascine et, quoique voilé, féconde ses paroles :

"Ne faut-il pas plutôt penser que même, sans être jamais vérifiables, elles nous portent vers ce qu'il peut y avoir autour de nous ou en nous de vérité cachée... " (9).

L'Illimité échappant à nos sens et à notre compréhension, un certain type d'image deviendra nécessaire pour que le poème puisse recueillir ne serait-ce que des échos de la profondeur voilée des choses. Et du moment qu'il s'impose de fondre les limites et l'Illimité, cette image aura pour mission d'élancer vers l'Absolu non pas à travers l'oubli ou la déformation des réalités, mais, paradoxalement, par une plus grande fidélité envers celles-ci. C'est vers les images « qui ne naissent pas du tout du désir (inconscient ou non) de fuir, de cacher ou d'altérer le premier terme de la comparaison " (10), que se dirige le poète.

L'œuvre de Jaccottet ne présente pas de prise de position purement théorique dans ce domaine; bien au contraire, l'image est elle-même bâtie sur cette tentation et sur ce refus. La simultanéité des deux réactions opposées du poète entraîne une méfiance, une rétention de l'essor métaphorique, qui ne trouve pas d'acquiescement complet. C'est comme si ces réflexions étaient sous-jacentes à la plupart des images :

"Méfie-toi des images. Méfie-toi des fleurs. Légères comme les paroles. Peut-on savoir si elles mentent, égarent ou si elles guident? Moi qui suis de loin en loin ramené à elles, moi qui n'ai qu'elles ou à peu près, je me mets en garde contre elles" (11).

La poésie de Jaccottet constitue une tentative de concilier les contraires, silence et parole, nomination directe et image, dans l'expression. La voix fluctue, penchant tantôt d'un côté, tantôt de l'autre, mais sans exclure 
aucun des opposés ; elle a pour fin ultime de dépasser les deux extrêmes et de les réunir en mettant à jour ce que les mots ne peuvent transmettre et qui, pourtant, est à l'origine de la création poétique.

La profonde bipôlarité qui tiraille l'être entre l'Absolu et l'ici-bas est rapidement transmise des conceptions générales à l'instrument mis en œuvre. Le poète tente de réaliser et de rendre par les mots les mouvements de l'essor et de la redescente, suivant la terminologie de Jean-Pierre Richard (12), qui relient les deux pôles. Essor et redescente qui tendent à se fondre en un mouvement unique de pénétration et de dépassement des choses, par désir de concilier les deux extrêmes. Mais, plus concrètement, c'est l'image qui est écartelée par cette double direction de la tentative poétique. Confronté à l'appel obscur que l'Illimité place au cœur de certaines réalités, le poète réagit fréquemment, malgré les risques du dénombrement, par les images. Et voilà que celles-ci, déjà " précaires " comme le dit Jean-Luc Seylaz, par la menace du silence pesant sur elles (13), doivent demeurer à la fois fidèles à la perception objective des choses, sinon la rendre plus pénétrante, et nous élever à l'« autre ", à l'inconnu que les apparences matérielles voilent.

Cette bipôlarité des images, qui tẹndent ainsi vers deux exigences apparemment irréconciliables, implique une profonde hẻtérogénéité dans leur nature, fondée sur une double démarche, rationnelle et irrationnelle. Nous voulons signifier par là les deux processus différents que Carlos Bousoño a mis à jour dans l'image traditionnelle et l'image contemporaine proprement dite, ou "visionnaire " dans sa propre terminologie. Processus essentiellement opposés par le rôle qui est accordé à la raison, qui entraînerait une perception logique et objective des réalités, et l'émotion, c'est-à-dire les associations irréfléchies de l'inconscient créateur (14). Les images traditionnelles se caractérisent par une démarche rationnelle de la part du poète qui les fonde sur des analogies évidentes, immédiatement perceptibles pour la raison, tandis que, toujours selon ce critique, les images visionnaires se caractérisent par des rapports émotionnels entre les réalités rapprochées, grâce aux associations inconscientes et communes qu'elles produisent.

La quête de l'Illimité, telle qu'elle est menée et par la nature particulière de celui-ci, est intimement liée à une démarche irrationnelle. Toutè quête de l'Absolu ne nécessite pas forcément une telle démarche : dans bien des conceptions du monde, l'Illimité constitue une évidence irréfutable et, de plus, les voies par lesquelles il se manifeste à l'homme sont solides. Rien de cela pour Jaccottet, car l'Absolu auquel il se voue n'est qu'une possibilité incertaine et le réseau épars dans la nature à travers lequel il devrait se manifester demeure lui-même fragile et douteux. Ni évident ni lisible, c'est dit, du moins par la parole logique et courante. L'usage de l'image intuitive ou irrationnelle découle de l'impossibilité éprouvée par le poète d'expliquer clairement la fragile expérience de 
l'« autre " qu'il lui arrive de subir parfois. L'indicible est d'ailleurs, d'après Michel le Guern, une des motivations essentielles de l'image, en particulier pour les mystiques :

«Dépasser par le langage ce que peut dire le langage de la toute simple information logique pour essayer de donner une information logique d'un ordre supérieur, voilà qui fournit, dans la poésie, dans le langage amoureux, une des motivations les plus pressantes au processus métaphorique " (15).

L'on pourrait rapprocher la poésie de Jaccottet d'une certaine mystique, encore faudrait-il préciser que l'expérience de l'Illimité n'aboutit pas chez celui-ci à une communion avec l'ordre transcendant, qui n'est peut-être que fausse illusion, mais à la tentative toujours recommencée d'y parvenir.

L'appel, fragile et douteux, frappe parfois le poète alors qu'il se trouve confronté à un objet ou un phénomène particuliers de la nature. Ce qui l'a ainsi interpelé ne pouvant être un reflet direct de l'Illimité, la seule issue qui se présente est de se laisser aller à cet appel, de l'explorer par l'intuition irréfléchie, par la rêverie : « on laisse venir, on laisse aller les images " (16), sans se forcer à saisir des rapports précis et évidents. A un moment donné, quand il se promenait, grâce à un mot qui lui est venu tout à coup à l'esprit, le mot « joie ", il a commencé à métamorphoser le cadre qui l'entourait. Puis, retraçant cette expérience, voilà comment il conclut :

« Alors, à la naissance hivernale d'une autre année [...] je me suis mis, non pas à réfléchir, mais à écouter et recueillir des signes, à dériver au fil des images ; comprenant, ou m'assurant paresseusement, que je ne pouvais faire mieux, quitte à n'en retenir après coup que des fragments, même imparfaits et peu cohérents... " (17).

L'abandon des restrictions rationnelles peut nous faire retenir seulement des fragments "imparfaits et peu cohérents ", mais les facultés rationnelles étant incapables de pourvoir à l'élancement, la seule tentative valable pour se rapprocher de ce que voilent les choses est celle de la dérive par l'imagination :

"Fleurs couleur bleue, bouches endormies, sommeil des profondeurs " (18).

Nous sommes bien face à une image visionnaire, semblable à celles que décrit Carlos Bousoño. Nous pouvons, tout au plus, mettre à jour entre ses deux plans des rapports inconscients, de nature émotionnelle, au moyen d'une analyse des associations irréfléchies qu'ils produisent. La bouche endormie est associée au silence, à une vague occultation d'un secret ou une vérité qu'on tait. Et c'est sur ce silence qu'insiste, d'une façon voilée à notre entendement, l'image : silence de la fleur qui ne s'ouvre pas, qui ne livre pas son secret. L'exploration de l'objet par la 
démarche irrationnelle n'a finalement abouti qu'à la constatation de son opacité, ce qui, de plus, fonde l'image par le biais du silence auquel renvoient les réalités mises ici en rapport.

Mais une quête de cette nature ne serait-elle pas incomplète pour qui cherche une perception directe et objective des choses ? La dérive dans l'imaginaire, ne finirait-elle pas par nous plonger dans le pur subjectif, dans l'irréel, et par effacer l'authentique mesure de l'ici-bas que le poète veut absolument conserver?

La démarche rationnelle est intimement liée pour le poète au desır de rester accroché à la réalité tangible. L'image traditionnelle est ainsi requise par la nécessité de ne pas fausser les objets sur lesquels elle s'applique. Des analogies étant perçues de façon évidente entre les plans mis en rapport, la fidélité exigée au réel ne court plus aucun danger. Il est frappant de trouver dans l'œuvre de Jaccottet, à côté des images caractéristiques d'une poésie contemporaine, qui répondent à des rapprochements émotionnels, d'autres images pleinement traditionnelles :

"Ce matin il y avait un miroir rond dans la brume, un disque argenté près de virer à l'or » (19).

La raison saisit immédiatement les rapports objectifs qui relient les deux plans, et de ce fait l'image est rendue transparente. Cependant, le poète risque de cette façon de se borner à redire les choses, à tourner autour d'elles sans ouvrir de passage vers l'inconnu, même si les analogies perçues sont moins évidentes :

\author{
"L'une à l'aube d'été \\ Dans l'air de plus en plus clair \\ scintille encore cette larme \\ ou faible flamme dans du verre " (20).
}

Ces deux types d'images n'ont rien en commun pour nous faire penser, au premier abord, que ce sont les créations d'un même poète. Comment est-il possible que dans une seule œuvre se retrouvent des procédés métaphoriques si éloignés dans leur conception ? Mais cette question en appelle une autre : comment est-il possible de vouloir, une fois que l'unité entre l'au-delà et l'ici-bas a été rompue, concilier les limites et l'Illimité ? Déchirement de l'image en deux aboutissements opposés et déchirement de l'être entre deux pôles constituent des faits parallèles.

Aussi bien la démarche rationnelle que la démarche irrationnelle sont objets de refus et de nécessité parce qu'une seule démarche ne manquerait pas de faire échouer la double exigence que le poète s'est fixée : l'accès à l'Illimité et l'enracinement dans les limites. Chaque démarche apporte des bienfaits à l'entreprise poétique, mais sans l'autre, elle demeure finalement impuissante. L'irrationnel pur nous ferait en quelque sorte décoller infailliblement du ras des choses, mais il nous mènerait à un degré tel d'irréel que nous perdrions tout contact avec les 
limites qu'impose l'ici-bas. Jaccottet s'attaque à l'image arbitraire, qui tire son éclat du rapprochement de ce qu'il y a de plus disjoint, sans aucune rigueur :

"C'est critiquer là, avec juste raison, la facilité excessive avec laquelle les images obéissent à nos désirs ; les enfants en inventent, à un certain âge, tous les jours ; les surréalistes en ont inondé la poésie moderne " (21).

Le refus de l'irrationnel absolu est évident dans l'allusion peu élogieuse aux surréalistes - et l'on se rappellera la défense du caractère arbitraire de l'image par André Breton -, de même que dans ce refus de se laisser aller aux désirs, à une subjectivité qui nous éloignerait de l'authentique réalité. Cela n'entraîne pas pour autant un retour à l'image tautologique, bâtie sur des ressemblances immédiatement constatables et qui n'apporte aucune profondeur à l'objet, en se limitant à le parer. Nous obtiendrions ainsi des images belles, séduisantes, mais closes sur elles-mêmes, impuissantes à nous aider pour franchir le pas. Si l'Illimité et ses signes épars étaient transparents, peut-être qu'en mettant tout simplement ceux-ci en relief l'élévation atteindrait son but, mais, comme nous l'avons déjà constaté, Jaccottet conçoit d'une façon bien différente l'Illimité.

Le poète renonce à ce caractère arbitraire mais, malgré cela, du moins est-il contraint de ne pouvoir se libérer d'une part d'ombre, d'un ensemble de significations irrationnelles qui lui échappent, dans ses propres mots :

« Je ne peux plus parler qu'à travers ces fragments pareils à des pierres qu'il faut soulever avec leur part d'ombre " (22).

Ce qui semble ici une contrainte peut pourtant presque devenir une exigence :

" Mais je sais que cette suggestion ne doit pas être abstraite de la chose, qu'elle doit rester à l'intérieur, donc cachée dans le texte. C'est à la condition d'être plus ou moins cachée qu'elle agit ; autrement, on n'a plus qu'une formule qui intéresse l'intelligence... $\gg(23)$.

La poésie se replie ainsi dans l'obscur, dans la nécessité de préserver le mystère, l'inconnu que voilent les réalités, par l'opacité des images vis-àvis de la raison, du " labyrinthe cérébral ", aussi desséchants et menteurs que l'irrationnel absolu. Il appert de tout ceci que le poète est constamment rejeté d'une démarche à l'autre. Mais puisqu'aucune des deux n'est valable à elle seule, pourquoi ne pas essayer de les concilier en bâtissant les images à la fois sur l'une et l'autre ? A l'encontre de ce que l'on pourrait penser, la pratique de Jaccottet démontre que le rationnel et l'irrationnel ne sont nullement incompatibles, une double perspective éclairante et obscurcissante apparaissant parfois dans ses images :

“Cette neige : hermine enfuie" (24).

L'élément central du plan imaginaire entretient des rapports évidents avec le plan réel, par le biais de leur couleur. Cependant, le second 
élément imaginaire, "enfuie ", se trouve en dehors de l'analogie qui assure ouvertement la saisie rationnelle de l'image et, de ce fait, l'obscurcit en quelque sorte. C'est comme si le poète avait réagi face à une excessive transparence poúr qu'ainsi, à la lecture, nous nous arrêtions, inquiétés par ce terme que rien d'objectif ne justifie à première vue. Sa présence n'obéit pas aux liens analogiques mais à la réaction émotionnelle commune que produisent les deux réalités : la neige et l'hermine apparaissent comme fuyantes parce que, soit sur le plan matériel soit sur le plan de la connaissance, nous ne pouvons pas les saisir. Il s'établit alors un paradoxe : ce qui semble le plus obscur est ce qui éclaire le sens profond de l'image, mais d'une façon voilée et en faisant appel en premier lieu à nos réactions intuitives. D'autre part, l'on constate les limites d'une telle démarche : l'appel à l'émotionnel et l'obscur pour mieux saisir l'objet dans sa profondeur et s'élancer n'aboutit qu'à révéler que cela est impossible. Autrement dit, l'obscurité de l'image nous ramène à l'obscurité de l'objet ; malgré la double démarche sur laquelle l'image est bâtie, elle met en évidence l'échec de cette entreprise poétique.

Il est aussi possible de contenir l'irrationnel par une énonciation déterminée qui, laissant dans l'hypothétique l'identification entre les deux plans, empêche en quelque sorte l'image d'être pleinement soudée :

"Nuit miroitante

Moment où l'on dirait

que la source même prend feu " (25).

Le plan imaginaire est si mystérieux qu'il est retenu par son introducteur : " on dirait ». L'atténuation de l'image obscure au moyen d'une telle énonciation indique qu'une méfiance, répondant à une démarche rationnelle, contrebalance l'excès d'intuition. Mais Jaccottet insiste aussi à plusieurs reprises sur l'effet desséchant d'une attention trop minutieuse :

"Déjà toutes sortes de choses, je ne peux plus les regarder, les aborder, parce que je les ai poursuivies et presque possédées. Il en sera de même de ce verger, peut-être, si j'insiste. Ainsi, devrais-je me réjouir qu'il s'éloigne, qu'il m'échappe, rapide lièvre des neiges " (26).

C'est tout ce qui échappe qui intéresse finalement le poète : les limites de la connaissance fécondent ses paroles : "Je ne m'occupe pas tellement, au fond, des qualités propres à l'arbre; comme s'y applique superbement Francis Ponge ", dit-il (27). Il ne s'agit pas de scruter à la loupe mais de dépasser l'objet et d'atteindre ce qu'il cache. Le chemin de l'irrationnel, de l'intuitif est le seul à pouvoir explorer l'autre face de la réalité, à " ouvrir les portes". Ce à quoi Jaccottet applique finalement ses efforts c'est « non plus à une comparaison entre deux réalités sensibles, concrètes, telles qu'écume et lingerie, plutôt, à une prolongation, à un approfondissement de la chose visible selon son sens obscur et en quelque sorte imminent " (27). Creuser le sens obscur du phénomène est à l'opposé de l'image traditionnelle, fondée sur des ressemblances évidentes et 
immédiatement décelables ; mais, parallèlement, l'enracinement dans la réalité réclame des rapports clairs et constatables entre les réalités mises en rapport.

L'image de Jaccottet est conflictuelle de par sa propre nature ; elle est bâtie sur deux ordres d'oppositions : sa nécessité et son refus, la démarche rationnelle et la démarche irrationnelle. Et c'est de ce sol mouvant qu'elle tire tout son éclat particulier. Instables par leur origine, les solutions entre ces contraires peuvent par moments pencher tout à fait d'un côté et évincer l'opposant, mais ce n'est pas le propre de la voix du poète. Les images affirmées sans l'ombre d'un doute, et celles qui répondent à une seule démarche ne sont pas les plus caractéristiques. C'est quand l'image intègre en elle-même la méfiance nourrie à son égard et combine une double démarche, logique et intuitive, qu'elle montre la profonde solidarité, dans cette œuvre, entre l'expression et les coordonnées essentielles de l'entreprise poétique.

\section{Evelio MIÑNANO}

NOTES

1) Ph. Jaccottet, L'Ignorant, Paris, Gallimard, 1958, rééd. in : Poésie 1946-1967, Poésie/Gallimard, 1971, p. 97.

2) ibid., p. 76.

3) Ph. Jaccottet, Pensées sous les nuages, Paris, Gallimard, 1983, p. 48.

4) L'Ignorant, op. cit., p. 58.

5) $\mathrm{Ph}$. Jaccottet, A travers un verger, Montpellier, Fata Morgana, 1975, rééd. Gallimard, 1984, p. 91. 114.

6) Ph. Jaccottet, La promenade sous les arbres, Lausanne, Mermod, 1957, rééd. 1961, p.

7) ibid., p. 42.

8) Ph. Jaccottet, La Semaison, Payot, 1963, rééd. Gallimard, 1971, p. 201.

9) La Promenade sous les arbres, op. cit., p. 82.

10) ibid., p. 82.

11) A travers un verger, op.c cit., p. 17.

12) Richard, Jean-Pierre, "Philippe Jaccottet ", in Onze études sur la poésie contemporaine, Paris, Seuil, 1964, rééd. 1981, pp. 315-319.

13) « L'image est donc précaire, ambiguë, pour ne pas trahir le presque rien, pour dire à la fois le rêve et l'hésitation du rêveur " (Seylaz, Jean-luc, Philippe Jaccottet, une poésie et ses enjeux, Lausanne, Ed. de l'Aire, 1982, p. 51. 
14) Bousono, Carlos, Teoria de la expression poética, Madrid, Gredos, 1952 ; El irracionalismo poético, Madrid, Gredos, 1977, p. 53.

15) Le Guern, Michel, Sémantique de la métaphore et de la métonymie, Paris, Larousse, 1973, p. 72.

16) Ph. Jaccottet, Paysages avec figures absentes, Paris, Gallimard, 1970, pp. 59-60.

17) Pensées sous les nuages, op. cit., p. 26.

18) Ph. Jaccottet, Airs, Paris, Gallimard, 1967, rééd. in : Poésie 1946-1967, Op. cit., p. 146.

19) Pensées sous les nuages, op. cit., p. 36.

20) Airs, op. cit., p. 135.

21) La promenade sous leś arbres, op. cit., p. 114.

22) Pensées sous les nuages, op. cit., p. 31.

23) Paysages avec figures absentes, op. cit., p. 62.

24) Airs, op. cit., p. 103.

25) ibid., p. 123.

26) A travers un verger, op. cit., p. 42.

27) Paysages avec figures absentes, op. cit., p. 66. 